\title{
Hybrid TAPP/TEP Repair of Bilateral Obturator and Inguinal Hernias
}

\author{
Michael Passeri, MD, Annabelle Teng, MD, Scott Belsley, MD \\ Department of Surgery, Mt. Sinai St. Luke's-Roosevelt Hospital Center, New York, NY, USA (all authors)
}

\begin{abstract}
Introduction: Obturator hernias represent a rare cause of small-bowel obstruction. When diagnosed on computed tomographic (CT) scan in a stable patient, timely surgical repair is indicated. Because of the absence of large-scale studies, the ideal operative approach has yet to be defined.

Case Description: A 75-year-old woman presented to the emergency department with an incarcerated left-side obturator hernia manifesting as a small-bowel obstruction. The hernia was diagnosed on CT scan, and the patient was taken to the operating room for laparoscopic reduction and repair. During surgery, a contralateral obturator hernia and bilateral indirect inguinal hernias were discovered. Hybrid transabdominal preperitoneal (TAPP) and total extraperitoneal (TEP) approaches were used to reduce and demonstrate the viability of the incarcerated bowel and then to repair all 4 defects with mesh. The procedure was well tolerated, and the patient was discharged uneventfully after the return of bowel function.
\end{abstract}

Discussion: This case provides an example of how a hybrid TAPP/TEP laparoscopic approach can be effective for visualizing and repairing an obturator hernia as well as an incidentally discovered inguinal hernia.

Key Words: Obturator hernia, TEP, TAPP, Mesh repair

Citation Passeri M, Teng A, Belsley S, Hybrid. TAPP/TEP repair of bilateral obturator and inguinal hernias. CRSLS e2015.00101. DOI: 10.4293/CRSLS.2015.00101.

Copyright (C) 2016 by SLS, Society of Laparoendoscopic Surgeons. This is an open-access article distributed under the terms of the Creative Commons Attribution-Noncommercial-ShareAlike 3.0 Unported license, which permits unrestricted noncommercial use, distribution, and reproduction in any medium, provided the original author and source are credited.

Address correspondence to: Michael Passeri, MD, Department of Surgery, Mt. Sinai St. Luke's-Roosevelt Hospital Center, 1000 Tenth Avenue, Second Floor, New York, NY 10019. Telephone: 212-523-6970, Fax: 212-523-6495, E-mail: mpasseri@chpnet.org

\section{INTRODUCTION}

Herniation of abdominal viscera through the obturator foramen represents a rare etiology of mechanical small-bowel obstruction (0.2-1.6\% of all cases) and an unusual type of abdominal wall hernia (0.05-1\% of all hernias). ${ }^{1}$ Although the obturator foramen is the largest foramen in the human body, a sturdy, fibrous membrane covers all but the most superior aspect. The resulting tunnel is referred to as the obturator canal and allows passage of the obturator artery, vein, and nerve between the pelvis and anterior thigh. The risk factors associated with obturator herniation mirror those that lead to laxity of the membrane and widening of the canal. These include female gender ( $7: 1$ female-to-male ratio), advanced age, and poor nutrition. ${ }^{2}$

The most common clinical presentation of obturator hernia is that of small-bowel obstruction of unclear etiology. Other findings, such as palpable mass, neuralgia, and medial thigh pain, exacerbated by exten- sion, abduction, and medial rotation (Howship-Romberg $\operatorname{sign}^{3}$ ), have been noted in a minority of cases and are rarely relied on for diagnosis. ${ }^{4}$ Despite best efforts at early detection, it is not unusual for these hernias to be discovered during an exploratory laparotomy in the setting of an acute abdomen. For those patients fortunate enough to present without signs of threatened bowel, the computed tomographic (CT) scan has become the gold standard of diagnosis, with a diagnostic accuracy of $90 \%$.

\section{CASE REPORT}

A 75-year-old woman presented to our emergency department describing crampy abdominal pain for 2 days. The pain was described as intermittently severe and was poorly localized. She also noted 7 episodes of nonbloody, nonbilious vomiting. She denied passage of stool or flatus in the preceding 48 hours. Her medical history was significant for atrial fibrillation, for which she was taking aspirin. 
Hybrid TAPP/TEP Repair of Bilateral Obturator and Inguinal Hernias, Passeri M et al., jisls.

On initial examination, vital signs were within normal reference ranges. The patient was found to have a soft, nondistended abdomen, diffusely tender to deep palpation, but without guarding or rebound tenderness. She had no palpable hernia, and the Howship-Romberg sign was negative. Laboratory results were significant only for leukocytosis of 15,800 cells $/ \mathrm{mL}$. A CT scan of the abdomen revealed a left-side obturator hernia (Figure 1).

The diagnosis was an incarcerated obturator hernia with associated obstruction. A nasogastric tube was placed, and the patient was taken to the operating room for hernia reduction and repair. Our team decided to use a laparoscopic approach with port placement to allow for a hybrid transabdominal preperitoneal (TAPP) and total extraperitoneal (TEP) procedure. We began with a 5-mm visual trocar entry technique at Palmer's point, with additional 5-mm ports placed above the anterior superior iliac spine bilaterally. Palmer's point, which is $3 \mathrm{~mm}$ below the left costal margin in the midclavicular line, 5 is our entry of choice in the setting of dilated intestine and bowel obstruction. Not only does the adjacent rib support the peritoneum, thereby providing a natural countertension, but it is also as far away as possible from the point of mechanical obstruction. We feel that this point of entry, combined with preoperative nasogastric decompression, helps to avoid visceral injury upon trocar insertion.

Upon entry into the abdomen, we visualized the left-side obturator hernia along with a loop of incarcerated smallbowel. This segment was reduced with gentle traction and judged to be viable (Figure 2).

The rest of the abdominal wall was then examined, revealing an obturator hernia on the contralateral side, as well as bilateral indirect inguinal hernias. We decided that attempt-

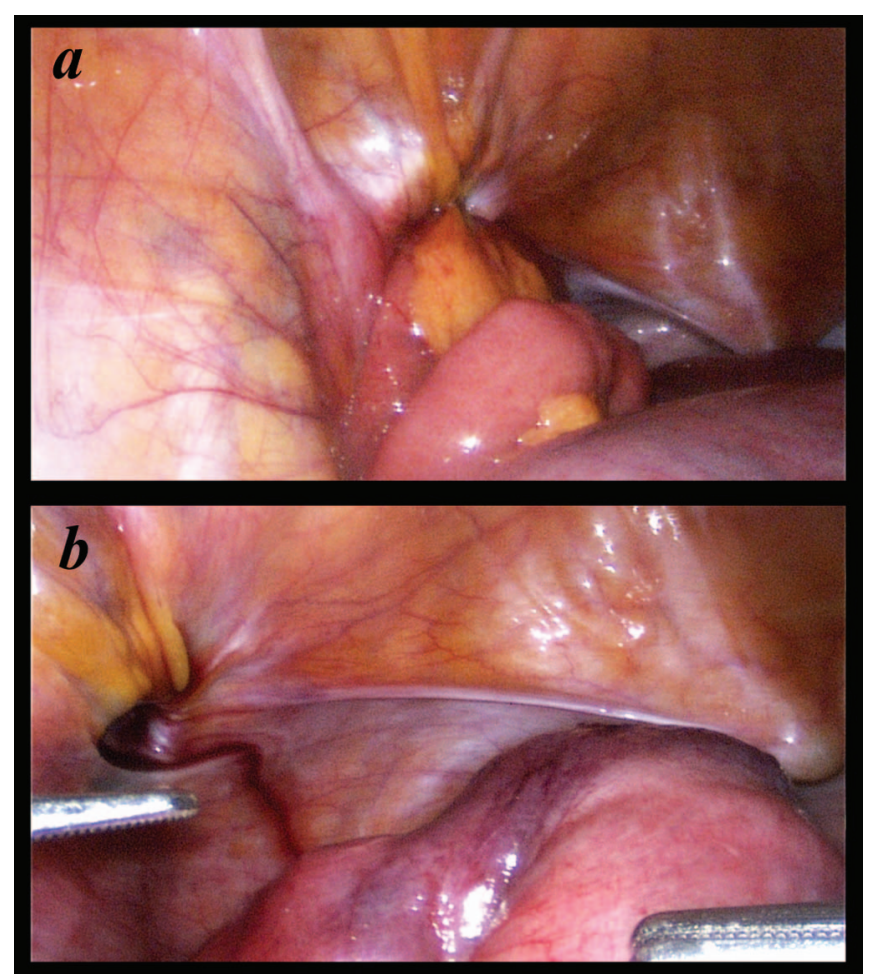

Figure 2. Left-side obturator hernia with incarcerated smallbowel before (A) and after (B) reduction. Some bruising is noted, but bowel appears viable.

ing to repair all hernias from our transabdominal vantage point would be difficult, because raising the requisite peritoneal flaps so low in the pelvis would provide a technical challenge. Therefore, we opted to convert to a TEP approach. Trocars were withdrawn, and the preperitoneal

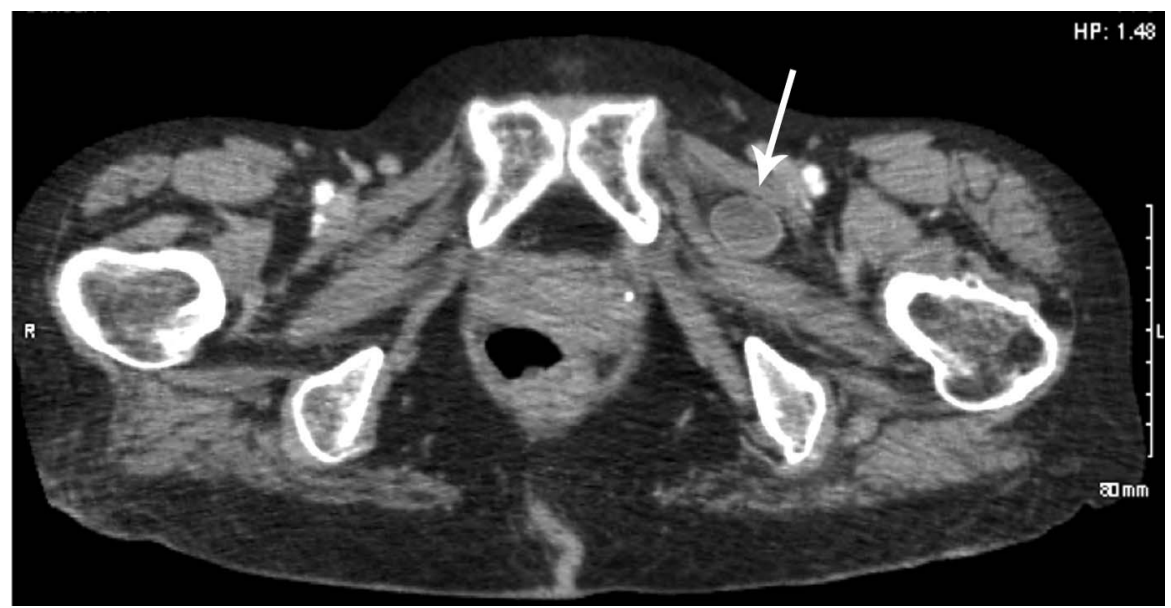

Figure 1. CT imaging revealing the location of a hernia sac between the obturator externus and pectineus muscles. Arrow: most common location for an obturator hernia. 


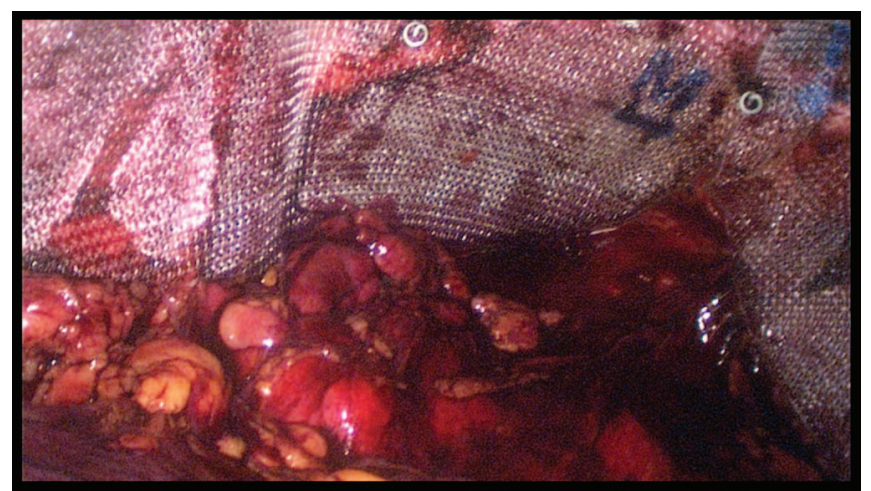

Figure 3. Extraperitoneal view of mesh placement over bilateral inguinal and obturator hernias. Tacks anchor mesh to the superior pubic rami.

space was expanded with a standard balloon technique through an umbilical incision. The trocars were then reintroduced, and dissection was performed caudad until adequate visualization of all 4 hernia defects was attained. A small amount of incarcerated fat was reduced from the right-side obturator hernia and the remainder of the hernia sac was reduced from the left side. Once adequate clearance had been obtained, we placed 2 pieces of polypropylene mesh such that all defects were completely covered (Figure 3). As we were working in the preperitoneal space, we noted an intact layer of peritoneum between the mesh and the intraabdominal viscera. The mesh was tacked to the pubic tubercle with a 5-mm ProTack fixation device (Covidien, North Haven, Connecticut), but tucked slightly lower than usual in the natural window between the bladder and the ischium. This position allowed for more adequate coverage of the obturator canal and the direct, indirect, and femoral spaces. After securing the mesh in place, we again withdrew all trocars and reinserted them into the peritoneal space. The abdomen was insufflated, and the previously reduced bowel was inspected for a final time. As before, it appeared viable. Before removing the camera, we took one last look at the peritoneum overlying the mesh and found it to be intact, with no mesh exposed.

\section{DISCUSSION}

Although obturator hernias are a rare cause of small-bowel obstruction, they should always be included in the working differential, especially in older female patients without a history of abdominal surgery. Often presenting without the classic findings of Howship-Romberg sign or obturator neuralgia, these hernias are frequently difficult to detect on physical examination. Our patient was a 75 -year-old woman who presented with small-bowel obstruction in the absence of obvious etiology and normal physical examination find- ings. CT imaging provided the diagnosis of an incarcerated left-side obturator hernia, prompting timely surgical intervention. We chose a combined TAPP/TEP approach, which allowed for broad visualization.

Much of the historic data from obturator hernia repair comes from emergent laparotomy in the setting of acute abdomen²; however, there is a growing body of literature that explores the nuances of a minimally invasive approach. ${ }^{6}$ Along with the general benefits of laparoscopy, including decreased postoperative pain and recovery time, this modality provides excellent visualization of the anterior abdominal wall, which, in our patient, meant discovery not only of a contralateral obturator hernia, but of bilateral inguinal hernias as well. With respect to the TAPP versus the TEP approach, it has been proposed that TAPP should be used when there is a question of a threatened bowel, as it allows for direct visualization and assessment of viability, whereas TEP should be reserved for decidedly elective cases. ${ }^{7}$ Our combination TAPP/TEP approach was instrumental in establishing bowel viability, defining all 4 hernias, and allowing secure mesh placement without the challenge of creating peritoneal flaps low in the pelvis.

\section{CONCLUSION}

Incarcerated obturator hernia is a rare and easily overlooked cause of small-bowel obstruction. When an obturator hernia is detected in a stable patient, timely surgical reduction and repair are indicated. In the reported patient, we demonstrated how a combined TAPP/TEP approach can be ideal for visualization of incarcerated bowel, exploring the abdominal wall for additional hernias, and employing mesh repair.

\section{References:}

1. Lo CY, Lorentz TG, Lau PW. Obturator hernia presenting as small bowel obstruction. Am J Surg. 1994;167:396-398.

2. Ziegler DW, Rhoads JE Jr. Obturator hernia needs a laparotomy, not a diagnosis. Am J Surg. 1995;170:67-68.

3. Karasaki T, Nakagawa T, Tanaka N. Obturator hernia: the relationship between anatomical classification and the HowshipRomberg sign. Hernia. 2004;18:413-416.

4. Yip AW, Ah Chong AK, Lam KH. Obturator hernia: a continuing diagnostic challenge. Surgery. 1993;113:266-269.

5. Agresta F, Mazzarolo G, Bedin N. Direct trocar insertion for laparoscopy. JSLS.2012;12:255-259.

6. Mantoo SK, Mak K, Tan TJ. Obturator hernia: diagnosis and treatment in the modern era. Singapore Med J. 2009;50:866-870.

7. Deeba S, Purkayastha S, Darzi A, Zacharakis E. Obturator hernias: a review of the laparoscopic approach. J Minim Access Surg. 2011;7:201-204. 